\title{
In vitro chemical stain removal by 'whitening' toothpastes
}

\author{
The chemical stain removal properties of 'whitening' toothpaste products: studies in vitro N. Sharif, E. MacDonald, \\ J. Hughes, R. G. Newcombe, and M. Addy Br Dent J 2000; 188: 620-624
}

\section{Background}

A considerable number of toothpastes are available as tooth whitening products. Most appear to contain ingredients that might remove extrinsic stains rather than change natural tooth colour. Extrinsic stain removal could be achieved by physical or chemical means.

\section{Aim}

The purpose of this study was to measure the chemical stain removal properties of a range of whitening toothpaste products and experimental formulations using a standardised method in vitro.

\section{Materials and method}

5 separate studies were conducted involving a total of 39 agents of which 28 were whitening products, 7 were experimental formulations, 2 were oxidising mouthrinses used as positive controls, 1 was a popular fluoride toothpaste product as a benchmark control, and 1 was water as the negative control. The formulations and controls varied in each study. The stain model was saliva/chlorhexidine/tea stain developed on optically clear acrylic to an optical density of at least 2.0. Groups of stained specimens were exposed to standard slurries or solutions of each test agent for 1 minute periods up to 5 minutes. Optical density readings were taken at each 1 minute time point. Analyses were based on per cent stain remaining after 5 minutes and time to $75 \%$ stain remaining.

\section{Results}

3 toothpaste products achieved $100 \%$ stain removal by 5 minutes; 2 of these in 3 out of 4 studies in which they were used. 4 experimental formulations also achieved $100 \%$ stain removal. In general agents with high total stain removal also had short times to $75 \%$ stain remaining. The majority of agents tested had low total chemical stain removal and prolonged times to $75 \%$ stain remaining. A few agents were little different from water and several similar in effect to the conventional fluoride toothpaste. This method in vitro tests agents under the best case scenario conditions for chemical stain removal.

\section{Conclusion}

Only a small number of the whitening toothpaste products have good chemical stain removal potential; the majority are unlikely to achieve their claimed benefits through chemical stain removal. There is clearly a need for further data on the actual effects of such products using both methods in vitro and particularly in vivo or in situ.

In brief

This paper:

- Details a simple in vitro method for comparing chemical stain removal by toothpastes.

- Provides a ranking of toothpastes to remove organic stains.

- Gives an understanding of the mechanism of staining associated with cationic antiseptics and metal salts.

- Summarises approaches to tooth whitening and the role of toothpastes.

- Considers the extrapolation of laboratory studies to clinical outcome.

\section{Comment}

xtrinsic stains on teeth can be removed Eby either physical or chemical means. This paper describes in vitro tests on the chemical stain removing properties of 28 commercially available 'whitening' toothpastes and 7 experimental formulations. Two oxidising mouthrinses provided positive controls, the negative control was water, and a family fluoride toothpaste was a benchmark control. Thirty-nine agents were tested altogether. None of the test toothpastes included bleaching agents at a level high enough to have an effect on tooth colour.

The tests used a standardised saliva/ chlorhexidine/tea stain developed on optically clear acrylic. Acrylic appears to stain in a similar manner to enamel and allows accurate optical density readings to be obtained. This stain model has been in use for many years, to investigate both stain formation and stain removal in vivo. In vitro, it provides a best case scenario for test agents because of the lack of potential for stain calcification. The stain removing effects of brushing are deliberately avoided. It should be noted that virtually all toothpastes have the potential for stain removal through abrasion.

Five experiments were carried out, using a standard slurry made by mixing 3 gm toothpaste/gel in $10 \mathrm{ml}$ water. Stained acrylic specimens were randomly allocated to each treatment, tumbled in the test slurry for 1 minute, rinsed in water and dried, before measuring the stain's optical density. The stain's progress was measured in two ways by the percentage of stain remaining after 1 to 5 minutes, and by the time needed to reduce the stain to $75 \%, 50 \%$ and $25 \%$. Since many of the formulations tested produced little effect, stain removal after 5 minutes and time to cut the stain to $75 \%$ were chosen as the most informative measures.
Two products, Aquafresh Whitening and Macleans Whitening, consistently showed 5-minute stain removal of $85-100 \%$. Super White and Rapid White Paste removed just over $80 \%$ of the stain. However, most toothpastes did not achieve $50 \%$ stain removal after 5 minutes and were little different from water in their effectiveness.

People want to have 'white' teeth and many claims are made for toothpastes. However, there has been a dearth of information about their comparative stain removing effectiveness. In this model, the majority of whitening toothpastes performed poorly, even toothpastes sold at considerably higher prices than normal family toothpastes; only two products come out of this test at all well.

\section{Diana Scarrott}

Head of Education and Science Department, British Dental Association 